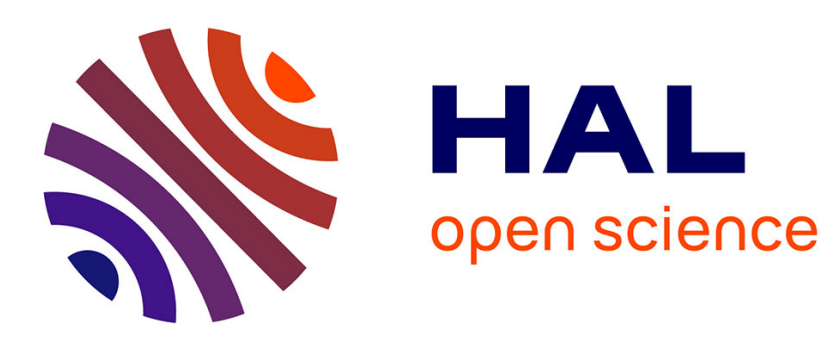

\title{
Two Monopole Antennas for Generating Radio OAM Waves in Circular Waveguide
}

\author{
W. Wei, K. Mahdjoubi, C. Brousseau, O. Emile, A. Sharaiha
}

\section{To cite this version:}

W. Wei, K. Mahdjoubi, C. Brousseau, O. Emile, A. Sharaiha. Two Monopole Antennas for Generating Radio OAM Waves in Circular Waveguide. EuCAP2016 - European Conference on Antennas and Propagation, Apr 2016, Davos, Switzerland. hal-01894320

\section{HAL Id: hal-01894320 \\ https://hal.science/hal-01894320}

Submitted on 12 Oct 2018

HAL is a multi-disciplinary open access archive for the deposit and dissemination of scientific research documents, whether they are published or not. The documents may come from teaching and research institutions in France or abroad, or from public or private research centers.
L'archive ouverte pluridisciplinaire HAL, est destinée au dépôt et à la diffusion de documents scientifiques de niveau recherche, publiés ou non, émanant des établissements d'enseignement et de recherche français ou étrangers, des laboratoires publics ou privés. 


\title{
Two Monopole Antennas for Generating Radio OAM Waves in Circular Waveguide
}

\author{
W. L. Wei ${ }^{1}$, K. Mahdjoubi ${ }^{1}$, C. Brousseau ${ }^{1}$, O. Emile $^{2}$, A. Sharaiha ${ }^{1}$ \\ ${ }^{1}$ Institute of Electronics and Telecommunications of Rennes (IETR), University of Rennes 1, Rennes, France \\ ${ }^{2}$ Laser Physics Laboratory (LPL), University of Rennes 1, Rennes, France \\ kouroch.mahdjoubi@univ-rennes1.fr
}

\begin{abstract}
We present a novel way to generate waves bearing Orbital Angular Momentum (OAM) in a circular waveguide by using two monopole antennas. The OAM mode $\ell=1$ is generated by a combination of the classical $T M_{01}$ and $T E_{21}$ modes of the circular waveguide. Two printed monopole antennas are used to create these two modes. The design procedure of this novel "OAM antenna" is presented. The resulting electrical field distribution and the radiation and phase patterns evidence the properties of waves bearing OAM.
\end{abstract}

Index Terms-orbital angular momentum, circular waveguide, monopole antenna.

\section{INTRODUCTION}

Orbital angular momentum (OAM) has been proposed to improve spectral efficiency [1-4] in radio communications, by creating multiple sub-channels of propagation corresponding to the twisting degree of the electromagnetic wave.

Whereas the phase of a usual plane wave is constant on the wave front, the phase $\alpha$ of OAM waves undergoes a linear variation along the angular coordinate $\varphi$ (roll angle): $\alpha=\ell \varphi$, where $\ell$ is an integer called the "topological charge" or the order of the OAM mode.

Up to now, in radio frequency bands, two main families of antennas have been proposed to generate OAM waves: circular phased arrays [1, 5-7] and plane wave transformers (spiral reflector, spiral phase plate and flat phase plate) [2, 8, 9].

In contrast, at optical frequencies different techniques are used to generate OAM beams [10]. As an example, a helical phased Laguerre-Gaussian mode $\mathrm{LG}_{01}(\ell=1$ or -1$)$ is obtained by combining two high-order Hermite-Gaussian modes of $\mathrm{HG}_{01}$ and $\mathrm{HG}_{10}$ [11]. Along this way, a rectangular silicon waveguide is already used to generate optical OAM beams by combining the $\mathrm{E}_{21}^{\mathrm{x}}$ and $\mathrm{E}_{12}^{\mathrm{x}}$ modes [12].

In the same manner, by combining some proper modes of a metallic waveguide we can create OAM waves in radio frequency bands.

In this paper, we combine the classical $\mathrm{TM}_{01}$ and $\mathrm{TE}_{21}$ modes of a circular metallic waveguide to generate radio OAM waves bearing a mode $\ell=1$. Two printed monopole antennas are introduced inside the circular waveguide to create the desired modes of $\mathrm{TE}_{21}$ and $\mathrm{TM}_{01}$.

\section{MODAL ANALYSIS}

The electrical field distributions of the $\mathrm{TM}_{01}$ and $\mathrm{TE}_{21}$ modes are reminded in Fig. 1.

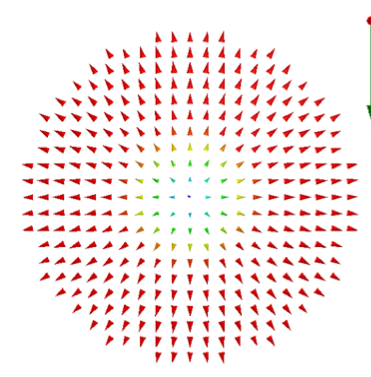

(a)

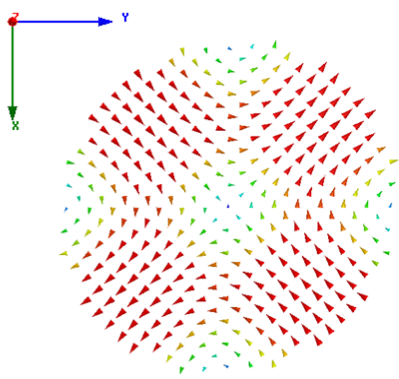

(b)
Fig. 1. Electrical field distributions of the classical modes in a circular waveguide: (a) $\mathrm{TM}_{01}$ mode; (b) $\mathrm{TE}_{21}$ mode

\section{A. $T M_{01}$ Mode}

The magnitude and phase patterns of $\mathrm{E}_{\mathrm{x}}$ and $\mathrm{E}_{\mathrm{y}}$ components for the $\mathrm{TM}_{01}$ mode are shown in Fig. 2. One can see that the magnitude of the $\mathrm{E}_{\mathrm{x}}$ component is minimal at the centre and in this respect; it is similar to an OAM wave. In fact, the beam of an OAM wave has a vortex with a minimum value of the field intensity in the centre and a linear phase rotation against the roll angle. For the $\mathrm{TM}_{01}$ mode, the phase of the $\mathrm{E}_{\mathrm{x}}$ component can be divided into two parts. For each part, the phase is constant on the wave front, and the phase difference between the two parts equals $\pi$. Due to the symmetrical distribution of the electrical field, the $E_{y}$ component has an identical distribution compared to the $\mathrm{E}_{\mathrm{x}}$ component, the only difference is that both the magnitude and phase patterns rotate $\pi / 2$.
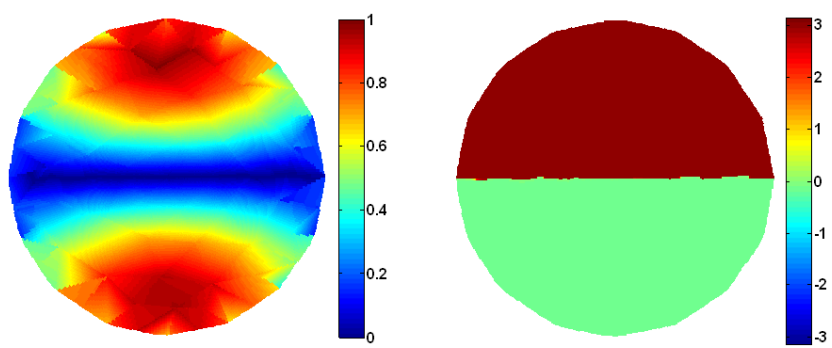

(a) 

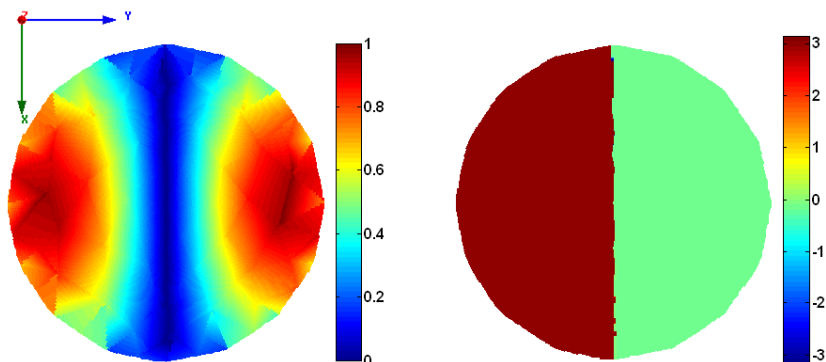

(b)

Fig. 2. Magnitude (left) and phase (right) patterns of the $\mathrm{TM}_{01}$ mode: (a) $\mathrm{E}_{\mathrm{x}}$ component; (b) $\mathrm{E}_{\mathrm{y}}$ component

\section{B. $T E_{21}$ Mode}

The magnitude and phase patterns of $\mathrm{E}_{\mathrm{x}}$ and $\mathrm{E}_{\mathrm{y}}$ components for the $\mathrm{TE}_{21}$ mode are shown in Fig. 3. We can observe that there is quite a similar distribution compared to the $\mathrm{TM}_{01}$ mode. The main difference is that for each component, there is a $\pi / 2$ rotation of both magnitude and phase patterns.
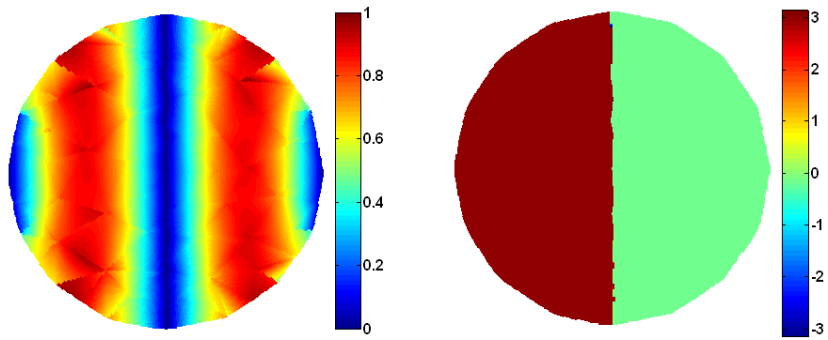

(a)
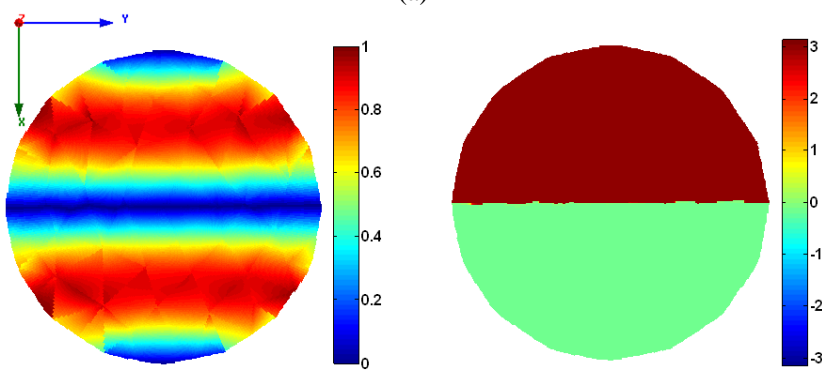

(b)

Fig. 3. Magnitude (left) and phase (right) patterns of the $\mathrm{TE}_{21}$ mode: (a) $\mathrm{E}_{\mathrm{x}}$ component; (b) $\mathrm{E}_{\mathrm{y}}$ component

\section{Combination of $T M_{01}$ and $T E_{21}$ Modes}

To create the OAM guided wave, we simultaneously excite the $\mathrm{TM}_{01}$ and $\mathrm{TE}_{21}$ modes with a $\pi / 2$ phase difference. The resulting magnitude and phase patterns of $E_{x}$ and $E_{y}$ components are shown in Fig. 4. We can observe that the magnitude patterns have a null at the center and the phases have a linear variation along the roll angle $\varphi$ with a $2 \pi$ phase shift in one turn. This shows indeed that for each component, both the amplitude and the phase correspond to a wave bearing an OAM mode $\ell$ equal to 1 .
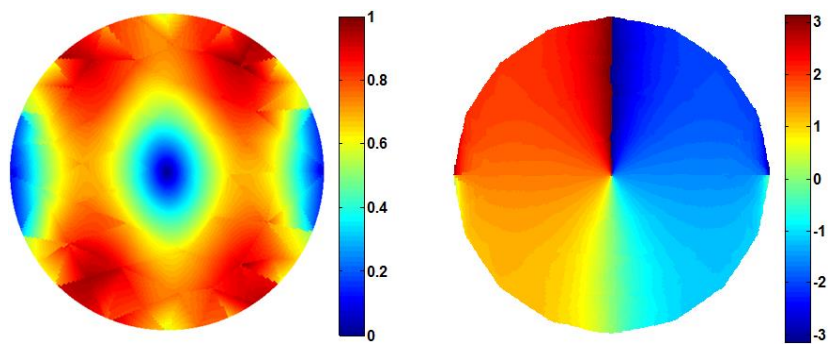

(a)
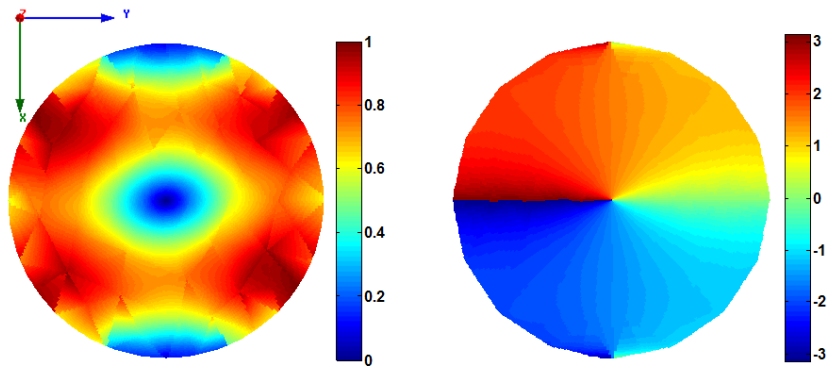

(b)

Fig. 4. Magnitude (left) and phase (right) patterns of the combined $\mathrm{TM}_{01}$ and $\mathrm{TE}_{21}$ modes: (a) $\mathrm{E}_{\mathrm{x}}$ component; (b) $\mathrm{E}_{\mathrm{y}}$ component

\section{ANTENNA DESIGN}

\section{A. Design Procedure}

We introduce two printed monopole antennas inside a circular metallic waveguide to generate the OAM waves. The configuration of the proposed "OAM antenna" is shown in Fig. 5 . The empty waveguide has a diameter of $4 \mathrm{~cm}$ and a height of $5 \mathrm{~cm}$, the corresponding cut-off frequencies of the $\mathrm{TM}_{01}$ and $\mathrm{TE}_{21}$ modes are respectively 5.7 and $7.3 \mathrm{GHz}$. We choose a working frequency of $8.3 \mathrm{GHz}$ to allow the propagation of the $\mathrm{TM}_{01}$ and $\mathrm{TE}_{21}$ modes and to prevent other high-order modes existing.

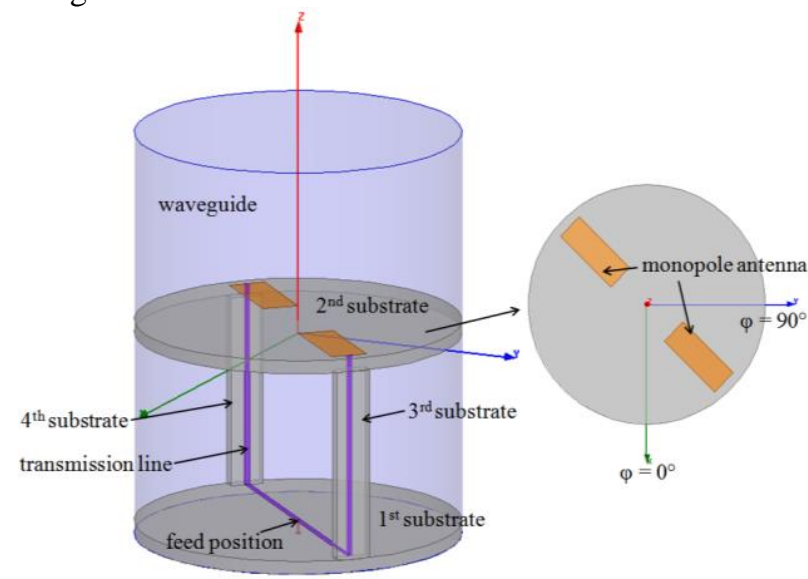

Fig. 5. Configuration of the proposed OAM antenna

From the electrical field distributions of the $\mathrm{TM}_{01}$ and $\mathrm{TE}_{21}$ modes (see Fig. 1); we can find that to create these two modes, the two monopole antennas should be placed on the same line and be out of phase. To this purpose, the two monopole antennas are connected with a single transmission line which is 
back-fed at the centre point with a $50 \Omega$ coaxial cable. In addition, since the field intensity of the $\mathrm{TE}_{21}$ mode is maximal at $\varphi= \pm 45^{\circ}$ and $\pm 135^{\circ}$ (see Fig. 1b), the monopole antennas should also be placed at the corresponding positions. In our design, we place the monopole antennas at $\varphi=45^{\circ}$ and $-135^{\circ}$.

The monopole antennas and the transmission line are both manufactured on FR4 substrates with a thickness of $1.6 \mathrm{~mm}$ and a relative permittivity of 4.4. The substrate totally has 4 parts. The $1^{\text {st }}$ substrate is placed at the bottom of the waveguide with the same diameter and the $2^{\text {nd }}$ one, in the middle of the waveguide. The $3^{\text {rd }}$ and $4^{\text {th }}$ substrates are placed along the side wall of the waveguide and are used to connect the $1^{\text {st }}$ and $2^{\text {nd }}$ substrates. The transmission line has a width of $0.7 \mathrm{~mm}$ and an impedance of $100 \Omega$. To satisfy the working frequency and the impedance matching, the length and width of each monopole antenna are optimized at 12 and $4.5 \mathrm{~mm}$, respectively.

\section{B. $\quad$ Simulation Results}

As depicted in Fig. 6, the antenna is well matched around 8.3 GHz.

The magnitude and phase patterns of the generated wave, observed on a plane perpendicular to the direction of propagation, are shown in Fig. 7. The observation window is a circular area with a radius of $3 \mathrm{~cm}$, lying $1 \mathrm{~cm}$ above the waveguide aperture. It can be seen that for both $E_{x}$ and $E_{y}$ components, the magnitude at the centre is much smaller than the surroundings and the phase rotates around the centre with a $2 \pi$ phase shift in one turn. This shows indeed that for each component, both the amplitude and the phase of the generated wave correspond to an OAM bearing wave with $\ell=1$.

At a working frequency of $8.3 \mathrm{GHz}$, the $\mathrm{TE}_{11}, \mathrm{TM}_{01}$ and $\mathrm{TE}_{21}$ modes are able to propagate in the waveguide. However, the monopole antennas are out of phase and due to this fact, the $\mathrm{TE}_{11}$ mode is eliminated. Thus, we can conclude that the OAM wave is generated by a combination of the $\mathrm{TM}_{01}$ and $\mathrm{TE}_{21}$ modes.

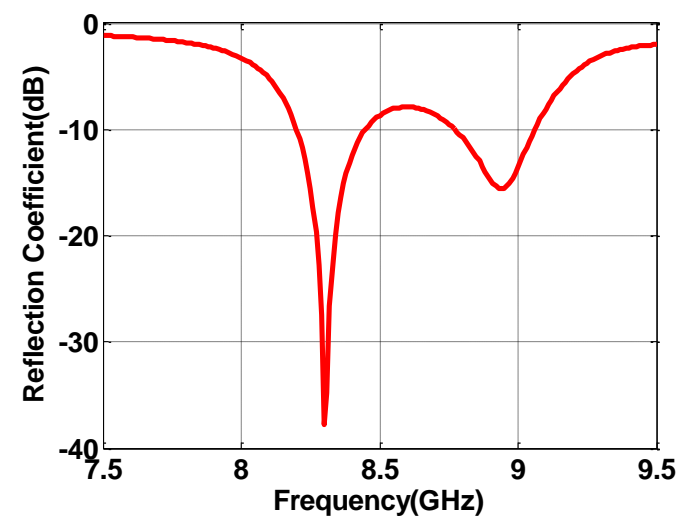

Fig. 6. Reflection coefficient of the OAM antenna
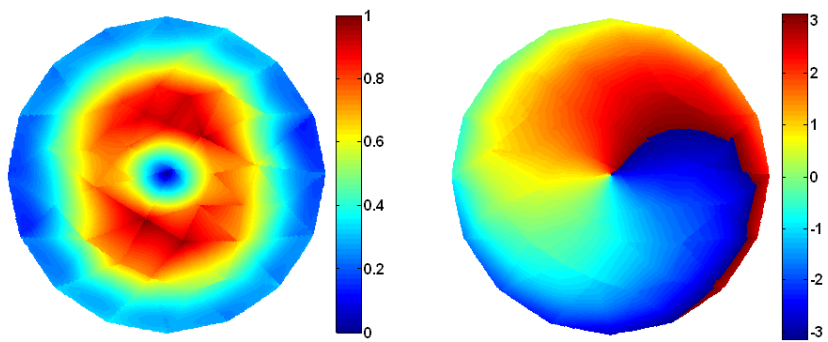

(a)
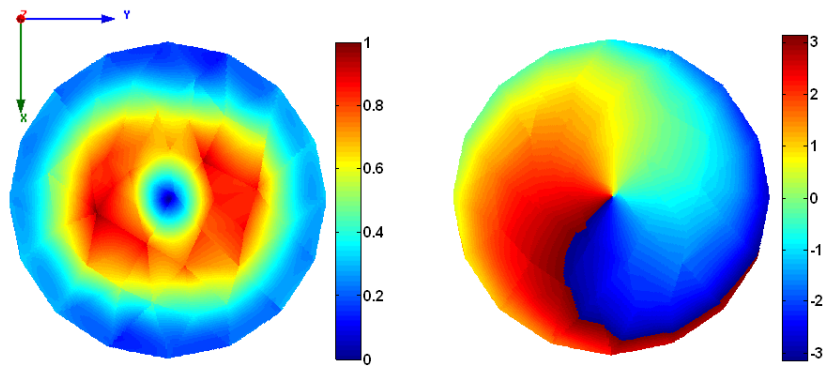

(b)

Fig. 7. Magnitude (left) and phase (right) patterns of the generated wave: (a) $\mathrm{E}_{\mathrm{x}}$ component; (b) $\mathrm{E}_{\mathrm{y}}$ component

Fig. 8 shows the $3 \mathrm{D}$ radiation and phase patterns of the OAM antenna. For simplicity, we just present the results of the $E_{\mathrm{x}}$ component. The radiation patterns in magnitude and phase depict the vortex that is characteristic of an OAM wave. The spiral form of the phase pattern, plotted in polar coordinates, corresponding to the linear variation of the phase against the roll angle ( $\varphi$, in Fig. 8b).

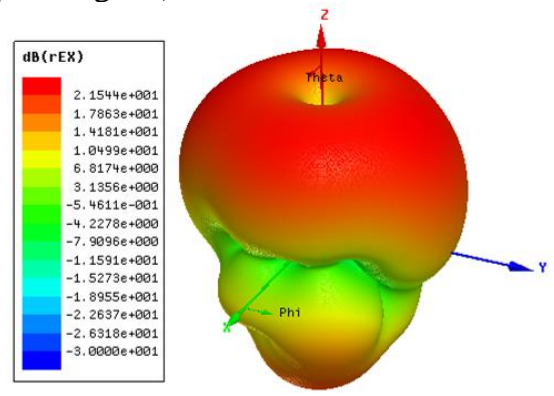

(a)

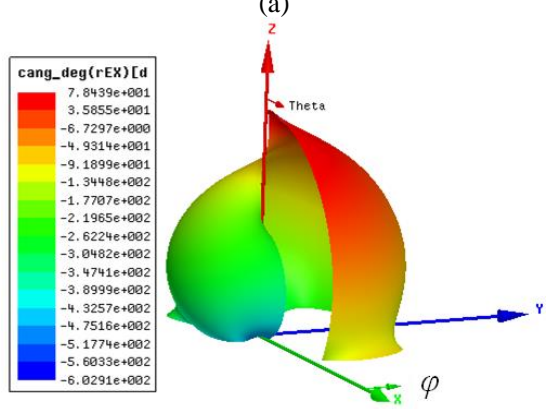

(b)

Fig. 8. 3D radiation and phase patterns of the OAM antenna: (a) magnitude; (b) phase 


\section{CONCLUSIONS}

In this paper, we have presented a method to generate radio OAM waves in a circular metallic waveguide by a combination of $\mathrm{TM}_{01}$ and $\mathrm{TE}_{21}$ modes. An antenna using two printed monopoles and a single transmission line is designed to create these two modes and hence, to generate an OAM wave with a topological charge $\ell=1$. Both the 2D magnitude \& phase patterns of the electrical field and the $3 \mathrm{D}$ radiation $\&$ phase patterns confirm the generation of an OAM bearing wave.

\section{ACKNOWLEDGMENT}

The authors would like to thank China Scholarship Council (CSC) (No. 201306090101) for scholarship support.

\section{REFERENCES}

[1] S.M. Mohammadi, L. Daldorff, J. Bergman, R. Karlsson, B. Thidé, K Forozesh, T. Carozzi, and B. Isham, "Orbital angular momentum in radio - a system study," IEEE Trans. Ant. Propag., vol. 58, no. 2, pp. 565-572, 2010

[2] F. Tamburini, E. Mari, A. Sponselli, B. Thidé, A. Bianchini, and F. Romanato, "Encoding many channels on the same frequency through radio vorticity: first experimental test," New. J. Phys., vol. 14, pp. 033001, 2012.

[3] J. Wang, J.Y. Yang, N. Ahmed, S. Dolinar, and A.E. Willner, "Terabit free-space data transmission employing orbital angular momentum multiplexing," Nat. Photon., vol. 6, pp. 488-496, 2012.
[4] Y. Yan, G.D. Xie, N. Ahmed, M. Tur, and A.E. Willner, "High-capacity millimetre-wave communications with orbital angular momentum multiplexing," Nat. Commun., vol. 5, 4876, 2014.

[5] B. Thidé, H. Then, J. Sjöholm, K. Palmer, J. Bergman, T.D. Carozzi, Ya.N. Istomin, N.H. Ibragimov, and R. Khamitova, "Utilization of photon orbital angular momentum in the low-frequency radio domain," Phys. Rev. Lett., vol. 99, pp. 087701, 2007.

[6] Q. Bai, A. Tennant, and B. Allen, "Experimental circular phased array for generating OAM radio beams," IET Electron. Lett., vol. 50, no. 20, pp. 1414-1415, 2014.

[7] W.L. Wei, K. Mahdjoubi, C. Brousseau, and O. Emile, "Generation of OAM waves with circular phase shifter and array of patch antennas," IET Electron. Lett., vol. 51, no. 6, pp. 442-443, 2015.

[8] G.A. Turnbull, D.A. Robertson, G.M. Smith, L. Allen, and M.J. Padgett, "The generation of free-space Laguerre-Gaussian modes at millimeterwave frequencies by use of a spiral phaseplate," Opt. Commun., vol. 127, pp. 183-188, 1996.

[9] R. Niemiec, C. Brousseau, K. Mahdjoubi, O. Emile, and A. Ménard, "Characterization of an OAM flat plate antenna in the millimeter frequency band," IEEE Antennas Wirel. Propag. Lett., vol. 13, pp. 1011-1014, 2014

[10] M. Padgett, and L. Allen, "Light with a twist in its tail," Contemp. Phys., vol. 41, no. 5, pp. 275-285, 2000.

[11] L. Allen, M. Beijersbergen, R. Spreeuw, and J. Woerdman, "Orbital angular momentum of light and the transformation of LaguerreGaussian laser modes," Phys. Rev. A, vol. 45, no. 11, pp. 8185-8189, 1992.

[12] D. Zhang, X. Feng, K. Cui, F. Liu, and Y. Huang, "Generating in-plane optical orbital angular momentum beams with silicon waveguides," IEEE Photon. J., vol. 5, no. 2, pp. 2201206, 2013. 